\title{
The acute effects of one session reactive neuromuscular training on balance and knee joint position sense in female athletes with dynamic knee valgus
}

\author{
Fatemeh Mirzaee, Rahman Sheikhhoseini*, and Hashem Piri \\ Faculty of Physical Education and Sport Sciences, Allameh Tabataba'i University, Tehran, Iran
}

Copyright: (C) 2020 F. Mirzaee et al. This is an open access article licensed under the Creative Commons Attribution License (https://creativecommons.org/licenses/by/4.0/).

\begin{abstract}
Background: Dynamic knee valgus (DKV) is defined as a combination of excessive femoral adduction, internal rotation, tibial internal torsion, and the medial movement of the knee. It appears that the athletes with DKV are at higher risk of developing knee injuries. Objective: The aim of this study was to evaluate whether the reactive neuromuscular training would improve whole-body dynamic balance and knee joint position sense (JPS) in women with the dynamic knee valgus. Methods: A controlled laboratory study was conducted on 28 female athletes with DKV. The single leg squat test was used to assess the DKV. All participants performed the reactive neuromuscular training, stationary cycling and resting sessions on three different days in a random order. The knee JPS and balance in the participants were measured immediately before and after the interventions. Kinematic analysis was used to measure the knee JPS in sagittal and frontal planes. In this regard, captured photos in the sagittal and frontal planes were analyzed using Kinovea software. The Y-balance test was applied to measure the balance. The data were analyzed by the repeated measures analysis of variance with a Bonferroni correction. Results: The results of the repeated measures analysis of variance showed that there was a significant difference between the sessions with respect to knee JPS in sagittal $(F(2,54)=3.323, p=.047)$ and frontal $(F(2,54)=23.83, p=.001)$ planes, respectively. Also, a significant difference in Y-balance test scores was observed between the sessions $(F(2,54)=9.12, p=.001)$. Conclusions: The results of this study showed that reactive neuromuscular training improved joint position sense and balance in individuals with DKV. The findings of this study may provide a basis for developing injury prevention and rehabilitation strategies in athletes with DKV.
\end{abstract}

Keywords: female athletes; reactive neuromuscular training; dynamic knee valgus; proprioception

\section{Introduction}

Lower extremity injuries are common in sports involving a high frequency of jump-landing movements (López-Valenciano et al., 2019; Mayer, Rühlemann, \& Jäger, 2019; Migliorini et al., 2019). Developing an efficient neuromuscular training program for the prevention and rehabilitation of knee injuries is one of the necessities of sports medicine research (Lee, Ren, Chang, Geiger, \& Zhang, 2014). These jumplanding movements are associated with ligamentous injuries like anterior cruciate ligament (ACL) sprains and Achilles tendonitis (Wertz, Galli, \& Borchers,

\footnotetext{
* Address for correspondence: Rahman Sheikhhoseini, Department of Corrective Exercise and Sport Injury, Faculty of Sport Sciences, Allameh Tabataba'i University, Western Azadi Sport Complex Boulevard, Hakim Highway, Tehran, Iran. E-mail: rahman.pt82@gmail.com
}

2013). The jump-landing movements can cause other non-ligamentous injuries such as bone and cartilaginous injuries (Aerts, Cumps, Verhagen, Verschueren, $\&$ Meeusen, 2013). The risk of injury may increase as a result of the faulty movement patterns (Padua et al., 2009). Dynamic knee valgus (DKV) as one of these faulty movement patterns is defined as a combination of excessive femoral adduction, internal rotation, tibial internal torsion and the medial movement of the knee (Lima et al., 2018). Movement patterns are modifiable risk factors for the injuries (Padua et al., 2009), and, the risk of ACL and other lower extremity injuries may be decreased by correcting faulty movement patterns.

One of the causes of faulty movement patterns may be impaired joint position sense (JPS) as a component of proprioception. The proprioception is a broad concept that includes both the sense of body orientation and position, and a sense of body and limb motion (Bragonzoni et al., 2019; Hillier, Immink, \& Thewlis, 
2015). It is a principal constituent of neuromuscular performance (Suner-Keklik, Cobanoglu-Seven, Kafa, Ugurlu, \& Guzel, 2017). Proprioception is the most important factor in balance, joint stability, coordination, and injury prevention (Bragonzoni et al., 2019). The proprioceptive impairment in the knee is related to injuries such as femoropatellar syndrome and ACL sprains (Ouattas, Wellsandt, Hunt, Boese, \& Knarr, 2019; Romero-Franco, Montaño-Munuera, FernándezDomínguez, \& Jiménez-Reyes, 2019).

In the high-risk sports such as handball, volleyball, basketball, and soccer, increased knee valgus or medial knee displacement due to weak neuromuscular control during dynamic movement tasks have been identified as the major risk factors for ACL injuries (Alentorn-Geli et al., 2009; Hewett et al., 2005; Krosshaug et al., 2016). It has been previously reported that core muscle activation, hip external rotators and abductors strength, and neuromuscular activation methods of the thigh muscles can play a major role in the reduction and prevention of the DKV (Ellenberger et al., 2020; Jamison, McNally, Schmitt, \& Chaudhari, 2013; Malloy, Morgan, Meinerz, Geiser, \& Kipp, 2016; Myer, Ford, \& Hewett, 2005; Stickler, Finley, \& Gulgin, 2015).

Reactive neuromuscular training (RNT) is considered as one of the methods of correcting the faulty movement patterns. The RNT program is designed to reestablish the joint dynamic stability and fine motor control (Guido \& Stemm, 2007). RNT has been used to rehabilitate low back pain, correct muscular imbalances, increase muscular flexibility, treat shoulder instability, decrease the risk of falling in people with Parkinson's disease, and improve the coordination and balance (Gray Cook \& Fields, 1999; Guido \& Stemm, 2007; Harrison \& Hart, 2011; Kim, 2012; Loutsch, Baker, May, \& Nasypany, 2015; Seada, Elsayed, \& Talat, 2013). Previous studies have shown that attendance at exercise training sessions can positively affect knee sense of position (Aslan, Buddhadev, Suprak, \& San Juan, 2018; David, Amasay, Ludwig, \& Shapiro, 2019) and this effect may be observable even in one session (Romero-Franco \& Jiménez-Reyes, 2017). Also, it is shown that sense of position may affect the dynamic balance (Onursal Kılınç et al., 2019) which may play a significant role in preventing sports injuries (Hanlon, Krzak, Prodoehl, \& Hall, 2020). To the best of our knowledge, no studies have been conducted on the effects of RNT on the position sense of knee and dynamic balance in female athletes with DKV.

Considering the high prevalence of DKV and its possible negative impact on performance among the female athletes; therefore, the aim of this study was to investigate the effects of RNT on dynamic balance and JPS of the knee in both frontal and sagittal planes, among athletes with DKV. In this study, the hypothesis was that the RNT would have positive effects on balance and JPS of the knee.

\section{Methods}

\section{Participants}

A controlled laboratory study was conducted on 28 female athlete volunteers, who met the inclusion and exclusion criteria (age 22.72 \pm 2.08 years, weight $57.1 \pm 5.5 \mathrm{~kg}$, height $165.1 \pm 6.55$, body mass index $\left.21.93 \pm 1.33 \mathrm{~kg} / \mathrm{m}^{2}\right)$. The study inclusion criteria were as follows: (i) females aged 19-24 years, (ii) having DKV based on single leg squat (SLS) test, and (iii) having at least 3 years of experience in amateur sports activities such as futsal, volleyball, or basketball. The subjects were excluded from the study if they had: (i) a history of knee surgery, (ii) a history of pathology, surgery or any other joint diseases in lower extremities and spinal column in last 5 years, (iii) any sensory-motor disorders, (iv) static postural abnormality such as static knee valgus based on New York postural assessment form, (v) body mass index lower than 18 or higher than 25, (vi) existence of pain in lower extremities and spinal column, or (vii) a history of neurological disorders or concussions that may affect their balance or ambulatory abilities. The study protocol was approved by the review board of the research ethics committee of the Faculty of Physical Education and Sport Sciences, Allameh Tabataba'i University, Tehran, Iran. During a familiarization session, the written informed consent was obtained from the participants, and their height, weight, and body mass index were recorded. All participants were assured that their personal information will be kept confidential, and they could withdraw from the study at any time.

\section{Protocols}

All data were collected in the biomechanics laboratory of Faculty of Physical Education and Sport Sciences, Allameh Tabataba'i University, Tehran, Iran. All participants were asked to come to the laboratory for three different days with a minimum interval of 48 hours between sessions. During each day, the kinematic data were first recorded as a pre-test measures, then the participants were asked to perform the predetermined protocol and immediately after that, the photogrammetric data were collected as the post-test measures. The three-day study protocol included a day of RNT training, a day of stationary cycling and a day of 30 minutes of resting on the table. The sequence of these protocols was selected in random order. All measurements were done by the same examiner. 


\section{DKV assessment}

The DKV was assessed using the SLS test. With their feet aligned in the sagittal plane and their arms folded across their chest, all participants performed five SLS repetitions of with the dominant leg. The leg used to kick a ball was considered to be dominant. Participants were trained to perform an SLS to 60 degrees of knee flexion and then return to the standing position. The movement was performed at a cadence of 2 seconds per descending phase and 2 seconds per ascending phase. We defined a positive SLS test as more than 3 abnormal responses on the dominant leg out of the 5 total trials. To learn the correct technique, all participants performed the task 2-3 times. The SLS has been reported as a valid and reliable test (Ageberg et al., 2010; Weeks, Carty, \& Horan, 2012). SLS test has been recommended as a reasonable tool to assess the DKV (Ugalde, Brockman, Bailowitz, \& Pollard, 2015).

\section{Knee proprioception measures}

Prior to testing, reflective markers (from reflective adhesive tapes with $10 \mathrm{~mm}$ of diameter) were positioned on the dominant leg of each participant, while the participants were asked to stand upright. In order to measure knee angle in the frontal plane, the markers were placed at on anterior superior iliac spine (ASIS), the midpoint of the line connecting the ASIS to mid patellae (mid-thigh), the midpoint of the line connecting lateral and medial malleolus (ankle), and the midpoint of the line connecting mid-malleolus to mid patellae (mid-shank). These marker placements were selected to eliminate the possible effect of patellar displacement on the knee valgus angle. The knee valgus angle was obtained as an acute angle that built from the intersection of a line connecting ASIS to mid-thigh with the line joining ankle and mid-shank markers.

In order to measure knee angle in the sagittal plane, markers were placed at the greater trochanter, lateral femoral epicondyle, and lateral malleolus. The knee flexion angle was obtained as an angle that built from the intersection of the line connecting greater trochanter to lateral femoral epicondyle with the line which connected lateral malleolus to lateral femoral epicondyle markers. All markers were placed by the same examiner.

Sixty degrees of knee flexion was considered as the test angle because muscles seem to have the greatest efficacy in the middle range of motion. In literature, 40 degree and 80 degree knee flexion are used as test angles (Larsen et al., 2005). A reliable technique for the assessment of knee position sense is a measurement of the replication of a specific target position, and the difference between the target and the estimated positions is considered as the outcome (Olsson et al., 2004). In this method, JPS is defined as the absolute error between the target and estimated positions (Larsen et al., 2005).

The JPS was measured using the photogrammetric method (Figure 1). To the knee JPS in a closed chain, the target angle was first drawn on the wall, then all participants performed the squat movement on the dominant leg with opened eyes. When participants reached sixty degrees of knee flexion, the pictures were taken by two cameras (Canon EOS 2000D, Canon, Ōta, Tokyo, Japan), one placed in the sagittal plane and the other in the frontal plane with 185 $\mathrm{cm}$ apart from the participants's place at the height of their knees. After performing 3 trials, to omit the

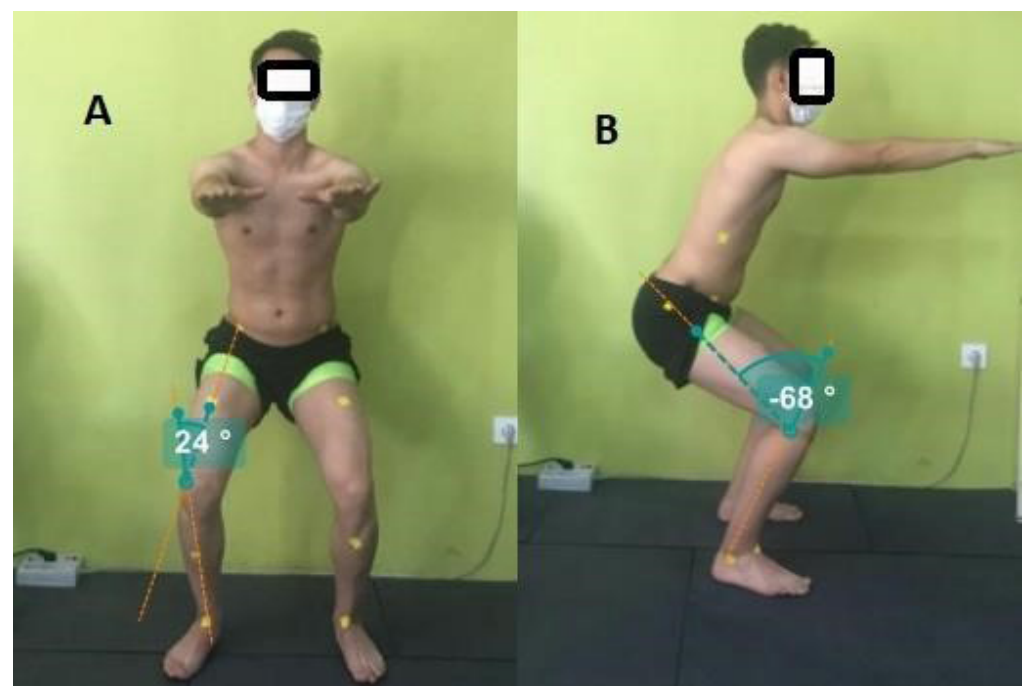

Figure 1. Calculating frontal (A) and sagittal (B) plane knee angles in the participants to examine knee sense of position. 
visual feedback during the measurement, the participant's eyes were closed using a band. Then each participant was asked to replicate the sixty degrees of knee flexion, stay in this position for 5 seconds, and memorize this angle. After 7 seconds of rest, the target angle was replicated by the participants again. Then the repositioning error was obtained by calculating the absolute values of subtracting the angles of closed eyes photos from angles of open eyes. These measures were done 3 times and the average repositioning error was used for further analysis. To obtain the effect of a specific protocol on repositioning error, the pre-post session changes of repositioning error were measured by subtracting the repositioning error of post-test from pre-test of each session.

\section{Dynamic balance measure}

To examine the dynamic balance, Y-balance test was performed based on the previous studies. The validity and reliability of the Y-Balance test have been reported in the previous studies (ICC $=.987,95 \%$ confidence interval [.972, .994]; Greenberg, Barle, Glassmann, \& Jung, 2019).

\section{Stationary bicycle training protocol}

After a 10-minute general warm-up, the participants trained for 30 minutes, using stationary bicycles. The height of the saddle was adjusted based on the ergonomic characteristics of the participants. They were asked to exercise at their preferred level of training intensity. At the end of the training session, cool down was done for 10 minutes.

\section{RNT training protocol}

RNT is a type of training designed to improve movement patterns through the active involvement of the participants in error detection and increasing feedbacks. These exercises must be performed with a minimum level of verbal feedback, aiming to worsen the faulty movement pattern of the individual (Gray Cook \& Fields, 1999). Thera bands were used to impose perturbational forces on participants. The intensity of training is defined based on the individual ability using the Borg scale. After a 10-minute warmup, the participants performed the RNT protocol for 40 minutes. To prevent the fatigue in participants, the intensity level of training was equal or less than 6 based on the Borg scale. In all exercises, the purpose was to increase knee valgus by the experimenter. Table 1 describes in more detail RNT program. In this study, a resting session was considered as a "control" condition.
Table 1

The RNT training protocol, including repetition or time for each exercise. The participants performed it one session per day separated from stationary cycling exercise. All exercises were performed for three sets.

\begin{tabular}{ll}
\hline Exercise & Repetition or time \\
\hline Single leg squat & $8-12$ \\
Wall squat & 10 \\
Romanian deadlift & 20 seconds, only with bodyweight \\
Lunge & 8 \\
Walking lunge & 8 \\
Side squat & 10 \\
Hurdle step & 10 \\
Jump squat & 10 \\
\hline
\end{tabular}

\section{Statistical analysis}

The data were analyzed using SPSS (Version 19; IBM, Armonk, NY, USA). The data normality was checked using the Shapiro-Wilk test. Mauchly's test of sphericity was used to examine the data sphericity. The data were then analyzed by the repeated measures analysis of variance with a Bonferroni correction. Fisher's LSD test was used to assess differences between the sessions. Partial eta squared was used as an effect size. All data analysis was done by considering $\alpha=.05$.

\section{Results}

The results from repeated measures analysis of showed that the knee JPS in the sagittal $(p=.047)$ and frontal $(p=.001)$ planes and Y-balance scores $(p=.001)$ significantly improved in RNT and SC sessions (Table 2 ). The post-hoc tests showed that there was a significant difference between RNT and stationary bicycle, and RNT and control condition in all study variables (Table 3).

\section{Discussion}

According to our results, the RNT program can improve the knee joint angle in the sagittal plane in female athletes with the DKV. The results of the current study are consistent with previous studies (Guido \& Stemm, 2007; Harrison \& Hart, 2011). In this regard, Guido and Stemm (2007) showed that an RNT program can re-establish the dynamic joint stability and neuromuscular control in patients with shoulder instability. Also, Harrison and Hart (2011) reported that RNT may 
Table 2

A comparison of changes in mean scores from the repeated measures analysis of variance test

\begin{tabular}{|c|c|c|c|c|c|c|c|}
\hline & \multicolumn{3}{|c|}{ Intervention $(M \pm S D)$} & \multirow[b]{2}{*}{$F(2,54)$} & \multirow[b]{2}{*}{$p$} & \multirow[b]{2}{*}{ Effect size } & \multirow[b]{2}{*}{ Power } \\
\hline & RNT & $\mathrm{SC}$ & Control & & & & \\
\hline \multicolumn{8}{|c|}{ Knee flexion (degrees) } \\
\hline Pre-session & $7.00 \pm 3.98$ & $6.71 \pm 4.23$ & $8.83 \pm 3.35$ & 3.32 & .047 & .104 & .594 \\
\hline Post-session & $2.91 \pm 1.48$ & $5.48 \pm 2.74$ & $6.82 \pm 3.40$ & & & & \\
\hline Mean change & $-4.09 \pm 3.29$ & $-1.22 \pm 5.66$ & $-2.01 \pm 3.66$ & & & & \\
\hline \multicolumn{8}{|c|}{ Knee valgus (degrees) } \\
\hline Pre-session & $6.82 \pm 3.40$ & $2.82 \pm 1.71$ & $3.83 \pm 2.31$ & 23.83 & .001 & .469 & 1.000 \\
\hline Post-session & $2.00 \pm 1.26$ & $2.91 \pm 1.64$ & $3.81 \pm 2.51$ & & & & \\
\hline Mean change & $-4.55 \pm 3.40$ & $-0.17 \pm 2.06$ & $0.02 \pm 3.17$ & & & & \\
\hline \multicolumn{8}{|c|}{ Y-balance (percents) } \\
\hline Pre-session & $84.66 \pm 10.99$ & $90.30 \pm 13.31$ & $90.29 \pm 11.27$ & 9.12 & .001 & .246 & .969 \\
\hline Post-session & $91.41 \pm 13.23$ & $91.75 \pm 11.55$ & $93.34 \pm 12.37$ & & & & \\
\hline Mean change & $6.76 \pm 5.26$ & $1.44 \pm 5.75$ & $3.06 \pm 4.27$ & & & & \\
\hline
\end{tabular}

Note. $\quad \mathrm{RNT}=$ reactive neuromuscular training; $\mathrm{SC}=$ stationary cycling.

Table 3

The results of Fisher's LSD and post-hoc tests in relation to joint position sense in sagittal and frontal planes and Y-balance score

\begin{tabular}{|c|c|c|c|}
\hline Pairwise comparison & $\begin{array}{l}\text { Difference of } \\
\text { means }\end{array}$ & $S D$ & $p$ \\
\hline \multicolumn{4}{|l|}{ Knee flexion(degrees) } \\
\hline RNT vs. SC & -2.86 & 1.31 & .038 \\
\hline RNT vs. Control & -2.86 & 0.82 & .018 \\
\hline SC vs. Control & -0.78 & 0.54 & .547 \\
\hline \multicolumn{4}{|l|}{ Knee valgus (degrees) } \\
\hline RNT vs. SC & 4.26 & 0.72 & .001 \\
\hline RNT vs. Control & 4.52 & 0.91 & .001 \\
\hline SC vs. Control & 0.20 & 0.76 & 0.76 \\
\hline \multicolumn{4}{|c|}{ Y-balance score (percents) } \\
\hline RNT vs. SC & 6.48 & 1.31 & .001 \\
\hline RNT vs. Control & 4.54 & 1.50 & .002 \\
\hline SC vs. Control & 1.93 & 1.81 & .209 \\
\hline
\end{tabular}

improve the patient-oriented outcomes in individuals with low back pain by restoring proprioceptive and kinesthetic sensory signals. RNT program is designed to diminish the need for audiovisual instruction from the athletic trainer. In RNT, the athlete responds to the external force created by an athletic trainer or physical therapist using an elastic tubing (Gray Cook \& Fields, 1999). The initial emphasis in RNT is on dynamic joint stability and proprioception (Gray Cook \& Fields, 1999). RNT can increase knee JPS by restoring the synergy and synchrony of muscle firing patterns required for dynamic joint stability and fine motor control (Voight \& Cook, 1996). Another explanation for the positive effect of RNT on knee JPS is the inherent ability of the exercise training to increase the cognitive appreciation of the joint's position and motion (Voight \& Cook, 1996).

RNT may augment the sensitivity of the muscle spindles and improve the knee JPS (Larsen et al., 2005). Accordingly, RNT diminishes the amount of error observed when measuring the JPS. In addition to the peripheral changes in receptors, some central changes such as the central facilitation of neural information, and increased somatosensory field of proprioception in the sensory cortex may result from RNT (Ashton-Miller, Wojtys, Huston, \& Fry-Welch, 2001; Lee et al., 2014).

Our results showed that RNT can improve knee proprioception in the knee valgus in female athletes with DKV. RNT may lead to an increased ratio of vastus medialis for vastus lateralis recruitment (Myer et al., 2005). Increased medial firing or decreased lateral firing can place the knee in an adducted position. It seems that RNT may alter the neuromuscular strategy in the knee region, in favor of better control of the knee. In this regard, another study showed that muscular activation can reduce the valgus laxity of the knee three-fold (Markolf, Graff-Radford, \& Amstutz, 1978). Others mentioned that peripheral and central adaptations could be considered as an explanation 
for the improvement in knee JPS in the frontal plane (Ashton-Miller et al., 2001; Larsen et al., 2005; Lee et al., 2014).

Reestablishment of dynamic joint stability is the primary purpose of the RNT program (Voight \& Cook, 1996). Through reestablishing dynamic joint stability, the control of abnormal joint translations like DKV could be possible during functional activities. RNT can increase postural awareness, kinesthesia, and knowledge of joint position, through increasing proprioception; therefore, RNT can decrease the risk of injury in the knee region.

Our results showed that RNT can improve the dynamic balance in female athletes with DKV. In this regard, the 8-week RNT is shown to improve balance ability (Kim, 2012). Moreover, another study reported that RNT could be considered as a useful and effective modality in the prevention of falls in people with Parkinson's disease (Seada et al., 2013). It has been proposed that RNT may improve muscular strength through neuromuscular changes (Gray Cook \& Fields, 1999). Also, the results of the current study showed that RNT may improve proprioception. It seems that both strength and proprioception may play an important role in promoting joint stability and balance maintenance (Blackburn, Guskiewicz, Petschauer, \& Prentice, 2000). Thus, these statements may provide some explanation for improving balance ability as the results of performing RNT. It is noteworthy to mention that our findings are unique and functionally important because JPS was measured under weightbearing conditions.

However, our study had some limitations. The study participants had DKV, so the result of this study could not be generalized to other populations. This study was conducted to investigate the immediate effect of RNT on the JPS and balance; therefore, further studies are necessary to examine the effect of long-term RNT on JPS and balance. Also, future research should investigate the durability of RNT effects. This method has a limitation of relying on memory instead of knee joint proprioception, too. The RNT training protocol includes several exercises and each exercise may activate several muscle groups, so, this variability in the exercises and activated muscles may affect the study results.

\section{Conclusions}

Our results showed that reactive neuromuscular training could improve joint position sense and balance in individuals with dynamic knee valgus. The findings of this study may provide a basis for developing injury prevention and rehabilitation strategies in athletes with dynamic knee valgus. Further studies are necessary to examine the durability and long-term effect of RNT on JPS and balance and possible effects of RNT on preventing some sports-related injuries in athletes.

\section{Acknowledgments}

The assistance provided by Dr. Ramin Balouchi was greatly appreciated.

\section{Conflict of interest}

There were no conflicts of interest.

\section{References}

Aerts, I., Cumps, E., Verhagen, E., Verschueren, J., \& Meeusen, R. (2013). A systematic review of different jumplanding variables in relation to injuries. Journal of Sports Medicine and Physical Fitness, 53, 509-519.

Ageberg, E., Bennell, K. L., Hunt, M. A., Simic, M., Roos, E. M., \& Creaby, M. W. (2010). Validity and inter-rater reliability of medio-lateral knee motion observed during a singlelimb mini squat. BMC Musculoskeletal Disorders, 11, 265.

Alentorn-Geli, E., Myer, G. D., Silvers, H. J., Samitier, G., Romero, D., Lázaro-Haro, C., \& Cugat, R. (2009). Prevention of non-contact anterior cruciate ligament injuries in soccer players. Part 1: Mechanisms of injury and underlying risk factors. Knee Surgery, Sports Traumatology, Arthroscopy, 17, 705-729.

Ashton-Miller, J. A., Wojtys, E. M., Huston, L. J., \& FryWelch, D. (2001). Can proprioception really be improved by exercises? Knee Surgery, Sports Traumatology, Arthroscopy, 9, 128-136.

Aslan, H., Buddhadev, H. H., Suprak, D. N., \& San Juan, J. G. (2018). Acute effects of two hip flexor stretching techniques on knee joint position sense and balance. International Journal of Sports Physical Therapy, 13, 846-859.

Blackburn, T., Guskiewicz, K. M., Petschauer, M. A., \& Prentice, W. E. (2000). Balance and joint stability: The relative contributions of proprioception and muscular strength. Journal of Sport Rehabilitation, 9, 315-328.

Bragonzoni, L., Rovini, E., Barone, G., Cavallo, F., Zaffagnini, S., \& Benedetti, M. G. (2019). How proprioception changes before and after total knee arthroplasty: A systematic review. Gait \& Posture, 72, 1-11.

David, E., Amasay, T., Ludwig, K., \& Shapiro, S. (2019). The effect of foam rolling of the hamstrings on proprioception at the knee and hip joints. International Journal of Exercise Science, 12, 343-354.

Ellenberger, L., Oberle, F., Lorenzetti, S., Frey, W. O., Snedeker, J. G., \& Spörri, J. (2020). Dynamic knee valgus in competitive alpine skiers: Observation from youth to elite 
and influence of biological maturation. Scandinavian Journal of Medicine \& Science in Sports, 30, 1212-1220.

Gray Cook, L. B., \& Fields, K. (1999). Reactive neuromuscular training for the anterior cruciate ligament-deficient knee: A case report. Journal of Athletic Training, 34, 194-201.

Greenberg, E. T., Barle, M., Glassmann, E., \& Jung, M.-K. (2019). Interrater and test-retest reliability of the Y balance test in healthy, early adolescent female athletes. International Journal of Sports Physical Therapy, 14, 204-213.

Guido, J. A., Jr., \& Stemm, J. (2007). Reactive neuromuscular training: A multi-level approach to rehabilitation of the unstable shoulder. North American Journal of Sports Physical Therapy, 2, 97-103.

Hanlon, C., Krzak, J. J., Prodoehl, J., \& Hall, K. D. (2020). Effect of injury prevention programs on lower extremity performance in youth athletes: A systematic review. Sports Health, 12, 12-22.

Harrison, B. C., \& Hart, J. M. (2011). Reactive neuromuscular training in low-back pain rehabilitation: Part two. Athletic Training and Sports Health Care, 3, 6-7.

Hewett, T. E., Myer, G. D., Ford, K. R., Heidt, R. S., Jr., Colosimo, A. J., McLean, S. G., ... Succop, P. (2005). Biomechanical measures of neuromuscular control and valgus loading of the knee predict anterior cruciate ligament injury risk in female athletes: A prospective study. American Journal of Sports Medicine, 33, 492-501.

Hillier, S., Immink, M., \& Thewlis, D. (2015). Assessing proprioception: A systematic review of possibilities. Neurorehabilitation and Neural Repair, 29, 933-949.

Jamison, S. T., McNally, M. P., Schmitt, L. C., \& Chaudhari, A. M. (2013). The effects of core muscle activation on dynamic trunk position and knee abduction moments: Implications for ACL injury. Journal of Biomechanics, 46, 2236-2241.

Kim, W.-W. (2012). Effects of reactive neuromuscular training and Baps-Trampoline training on balance ability and isokinetic muscle function. Official Journal of the Korean Academy of Kinesiology, 14, 127-136.

Krosshaug, T., Steffen, K., Kristianslund, E., Nilstad, A., Mok, K.-M., Myklebust, G., ... Bahr, R. (2016). The vertical drop jump is a poor screening test for ACL injuries in female elite soccer and handball players: A prospective cohort study of 710 athletes. American Journal of Sports Medicine, 44, 874-883.

Larsen, R., Lund, H., Christensen, R., Røgind, H., Danneskiold-Samsøe, B., \& Bliddal, H. (2005). Effect of static stretching of quadriceps and hamstring muscles on knee joint position sense. British Journal of Sports Medicine, 39, 43-46.

Lee, S. J., Ren, Y., Chang, A. H., Geiger, F., \& Zhang, L.-Q. (2014). Effects of pivoting neuromuscular training on pivoting control and proprioception. Medicine \& Science in Sports \& Exercise, 46, 1400-1409.

Lima, Y. L., Ferreira, V. M. L. M., de Paula Lima, P. O., Bezerra, M. A., de Oliveira, R. R., \& Almeida, G. P. L. (2018). The association of ankle dorsiflexion and dynamic knee valgus: A systematic review and meta-analysis. Physical Therapy in Sport, 29, 61-69.
López-Valenciano, A., Ruiz-Pérez, I., Garcia-Gómez, A., Vera-Garcia, F. J., Croix, M. D. S., Myer, G. D., \& Ayala, F. (2020). Epidemiology of injuries in professional football: A systematic review and meta-analysis. British Journal of Sports Medicine, 54, 711-718.

Loutsch, R. A., Baker, R. T., May, J. M., \& Nasypany, A. M. (2015). Reactive neuromuscular training results in immediate and long term improvements in measures of hamstring flexibility: A case report. International Journal of Sports Physical Therapy, 10, 371-377.

Malloy, P., Morgan, A., Meinerz, C., Geiser, C. F., \& Kipp, K. (2016). Hip external rotator strength is associated with better dynamic control of the lower extremity during landing tasks. Journal of Strength and Conditioning Research, 30, 282-291.

Markolf, K. L., Graff-Radford, A., \& Amstutz, H. (1978). In vivo knee stability. A quantitative assessment using an instrumented clinical testing apparatus. Journal of Bone and Joint Surgery, 60, 664-674.

Mayer, C., Rühlemann, A., \& Jäger, M. (2019). Verletzungen und deren Prävention beim Handball [Handball injuries and their prevention]. Der Orthopäde, 48, 1036-1041.

Migliorini, F., Rath, B., Tingart, M., Niewiera, M., Colarossi, G., Baroncini, A., \& Eschweiler, J. (2019). Injuries among volleyball players: A comprehensive survey of the literature. Sport Sciences for Health, 15, 281-293.

Myer, G. D., Ford, K. R., \& Hewett, T. E. (2005). The effects of gender on quadriceps muscle activation strategies during a maneuver that mimics a high ACL injury risk position. Journal of Electromyography and Kinesiology, 15, 181-189.

Olsson, L., Lund, H., Henriksen, M., Rogind, H., Bliddal, H., \& Danneskiold-Samsøe, B. (2004). Test-retest reliability of a knee joint position sense measurement method in sitting and prone position. Advances in Physiotherapy, 6, 37-47.

Onursal Kılınç, Ö., Ayvat, E., Ayvat, F., Sütçü, G., Kılınç, M., Aksoy, S., \& Aksu Yıldırım, S. (2019). The relationship between trunk position sense and postural control in ataxic individuals. Gait \& Posture, 68, 258-263.

Ouattas, A., Wellsandt, E., Hunt, N. H., Boese, C. K., \& Knarr, B. A. (2019). Comparing single and multi-joint methods to detect knee joint proprioception deficits post primary unilateral total knee arthroplasty. Clinical Biomechanics, 68, 197-204.

Padua, D. A., Marshall, S. W., Boling, M. C., Thigpen, C. A., Garrett, W. E., Jr., \& Beutler, A. I. (2009). The Landing Error Scoring System (LESS) is a valid and reliable clinical assessment tool of jump-landing biomechanics: The JUMP-ACL study. American Journal of Sports Medicine, 37, 1996-2002.

Romero-Franco, N., \& Jiménez-Reyes, P. (2017). Effects of warm-up and fatigue on knee joint position sense and jump performance. Journal of Motor Behavior, 49, 117-122.

Romero-Franco, N., Montaño-Munuera, J. A., FernándezDomínguez, J. C., \& Jiménez-Reyes, P. (2019). Validity and reliability of a digital inclinometer to assess knee joint position sense in an open kinetic chain. Journal of Sport Rehabilitation, 28, 332-338.

Seada, Y., Elsayed, E., \& Talat, W. (2013). Impact of reactive neuromuscular training on falling in Parkinson's disease. 
Indian Journal of Physiotherapy \& Occupational Therapy, 7, 65-70.

Stickler, L., Finley, M., \& Gulgin, H. (2015). Relationship between hip and core strength and frontal plane alignment during a single leg squat. Physical Therapy in Sport, 16, $66-71$.

Suner-Keklik, S., Cobanoglu-Seven, G., Kafa, N., Ugurlu, M., \& Guzel, N. A. (2017). The validity and reliability of knee proprioception measurement performed with inclinometer in different positions. Journal of Sport Rehabilitation, 26, 10.

Ugalde, V., Brockman, C., Bailowitz, Z., \& Pollard, C. D. (2015). Single leg squat test and its relationship to dynamic knee valgus and injury risk screening. Physical Medicine and Rehabilitation, 7, 229-235.

Voight, M. L., \& Cook, G. (1996). Clinical application of closed kinetic chain exercise. Journal of Sport Rehabilitation, 5, 25-44.

Weeks, B. K., Carty, C. P., \& Horan, S. A. (2012). Kinematic predictors of single-leg squat performance: A comparison of experienced physiotherapists and student physiotherapists. BMC Musculoskeletal Disorders, 13, 207.

Wertz, J., Galli, M., \& Borchers, J. R. (2013). Achilles tendon rupture: Risk assessment for aerial and ground athletes. Sports Health, 5, 407-409. 